\title{
Role of model ingredients on the directed flow and its disap- pearance using isospin dependent quantum molecular dynam- ics model
}

\author{
Rajni Bansal ${ }^{1, a}$ \\ ${ }^{1}$ Department of Physics, Panjab University, Chandigarh -160 014, India
}

\begin{abstract}
We study the effect of different equations of state, momentum dependence of nuclear forces and in-medium nucleon-nucleon cross-sections on the directed flow and its disappearance. Our findings reveal that soft momentum-dependent equation of state along with reduced cross-section shows good agreement with the experimentally observed mass dependence of balance energy.
\end{abstract}

\section{Introduction}

The collective transverse flow in heavy ion collisions has been used extensively over the past three decades to gain insight into the properties of nuclear matter at different thermodynamical conditions [1-3]. The dependence of transverse flow to various entrance channel parameters like incident energy [4], system size [2], colliding geometry [5, 6], mass asymmetry [7] and isospin of the reacting nuclei $[5,8]$ is well established experimentally as well as theoretically. The energy dependence of transverse flow, in particular is of great importance and it leads to a term known as balance energy $\left(\mathrm{E}_{b a l}\right)$ or energy of vanishing flow (EVF), where attractive and repulsive interaction counterbalance each other [9]. The balance energy is of great significance because experimentally determined balance energy can be easily compared with various theoretical calculations as it is free from any experimental uncertainties. A lot of efforts are being done in this direction and experimentally balance energies for more than 16 systems in mass range of 24-394 have been measured [10, 11]. Most of the measurements are for the semi-central collisions, only few are extended for the whole range of the colliding geometry [5, 11]. Due to extensive available data on balance energy, the corresponding theoretical efforts have also been carried out, but most of the theoretical attempts are limited to a much narrower range [12]. Sood et.al for the first time, carried out a complete and systematic study of mass dependence of balance energy for the reactions ranging from ${ }^{12} \mathrm{C}+{ }^{12} \mathrm{C}$ to ${ }^{238} \mathrm{U}+{ }^{238} \mathrm{U}$ using isospin-independent quantum molecular dynamics (QMD) model [2], but isospin degree of freedom also has good influence on the balance energy [5, 8]. Recently, Kumar et al. [13] studied mass dependence of balance energy using isospindependent quantum molecular dynamics (IQMD) model but their study was restricted to the mass range between $122\left({ }^{64} \mathrm{Zn}+{ }^{58} \mathrm{Ni}\right)$ to $394\left({ }^{197} \mathrm{Au}+{ }^{197} \mathrm{Au}\right)$ units. Thus, the complete and systematic study of mass dependence of balance energy using IQMD model is still missing.

ae-mail: rajnijain88@gmail.com

This is an Open Access article distributed under the terms of the Creative Commons Attribution License 2.0, which permits unrestricted use, distribution, and reproduction in any medium, provided the original work is properly cited. 
Here, we aim to understand role of nucleon-nucleon cross-section, different equations of state as well as the momentum dependence of the mean field and cross-section on the incident energy dependence of the directed flow for the four symmetric systems ${ }^{12} \mathrm{C}+{ }^{12} \mathrm{C},{ }^{40} \mathrm{Ca}+{ }^{40} \mathrm{Ca},{ }^{58} \mathrm{Ni}+{ }^{58} \mathrm{Ni}$ and ${ }^{197} \mathrm{Au}+{ }^{197} \mathrm{Au}$ covering the lighter and heavier system at impact parameter of $\hat{b}=0.4-0.6$. We also study the role of above mentioned quantities on mass dependence of balance energy for the reactions over the whole mass, ranging from ${ }^{12} \mathrm{C}+{ }^{12} \mathrm{C}$ to ${ }^{197} \mathrm{Au}+{ }^{197} \mathrm{Au}$ using isospin-dependent quantum molecular dynamics model.

The Isospin-dependent Quantum Molecular Dynamics Model (IQMD) [14] model has been used extensively for studying the isospin effects on large number of observables [8, 13].

\section{Results and Discussion}

For the present study, We simulate the reactions of ${ }^{12} \mathrm{C}+{ }^{12} \mathrm{C},{ }^{40} \mathrm{Ca}+{ }^{40} \mathrm{Ca},{ }^{58} \mathrm{Ni}+{ }^{58} \mathrm{Ni}$ and ${ }^{197} \mathrm{Au}+{ }^{197} \mathrm{Au}$ at incident energies of 50,100, 400, $800 \mathrm{MeV} / \mathrm{A}$ and at reduced impact parameter $\hat{b}=0.4-0.6$ using hard, soft and soft momentum-dependent (SMD) equation of the state (EOS) with free crosssection. Above Reactions are also simulated for SMD equation of state with reduced cross-section $\left(\sigma=0.8 \sigma_{\text {free }}\right)$ to see the role of cross-section. The reactions are followed till transverse in-plane flow saturates. The saturation time varies between $100 \mathrm{fm} / c$ (for lighter colliding nuclei such as ${ }^{12} \mathrm{C}+{ }^{12} \mathrm{C}$ ) and $300 \mathrm{fm} / c$ (for heavier colliding nuclei such as ${ }^{197} \mathrm{Au}+{ }^{197} \mathrm{Au}$ ). For the transverse flow we use the quantity "directed transverse momentum $\left\langle p_{x}^{\text {dir }}\right\rangle$ ", which is defined as: $\left\langle p_{x}^{\text {dir }}\right\rangle=\frac{1}{A} \sum_{i=1}^{A} \operatorname{sign}\{y(i)\} p_{x}(i)$, where $y(i)$ is the rapidity and $p_{x}(i)$ is the transverse momentum of $i^{\text {th }}$ particle.

In Fig. 1, we display the $\left\langle p_{x}^{d i r}\right\rangle$ as a function of incident energy for the reaction of ${ }^{12} \mathrm{C}+{ }^{12} \mathrm{C}$ (Fig. 1(a)), ${ }^{40} \mathrm{Ca}+{ }^{40} \mathrm{Ca}$ (Fig. 1(b)), ${ }^{58} \mathrm{Ni}+{ }^{58} \mathrm{Ni}$ (Fig. 1(c)) and ${ }^{197} \mathrm{Au}+{ }^{197} \mathrm{Au}$ (Fig. 1(d)). Circles, triangles, squares and open squares represent the calculations using soft, hard and SMD EOS with free crosssections, and SMD EOS with reduced cross-section $\left(\sigma=0.8 \sigma_{\text {free }}\right)$ respectively. It is evident from the figure that for all the cases and also at all incidents energies, SMD EOS with free cross section leads to highest value of $\left\langle p_{x}^{d i r}\right\rangle$ compared to all other options. As we already know from the literature

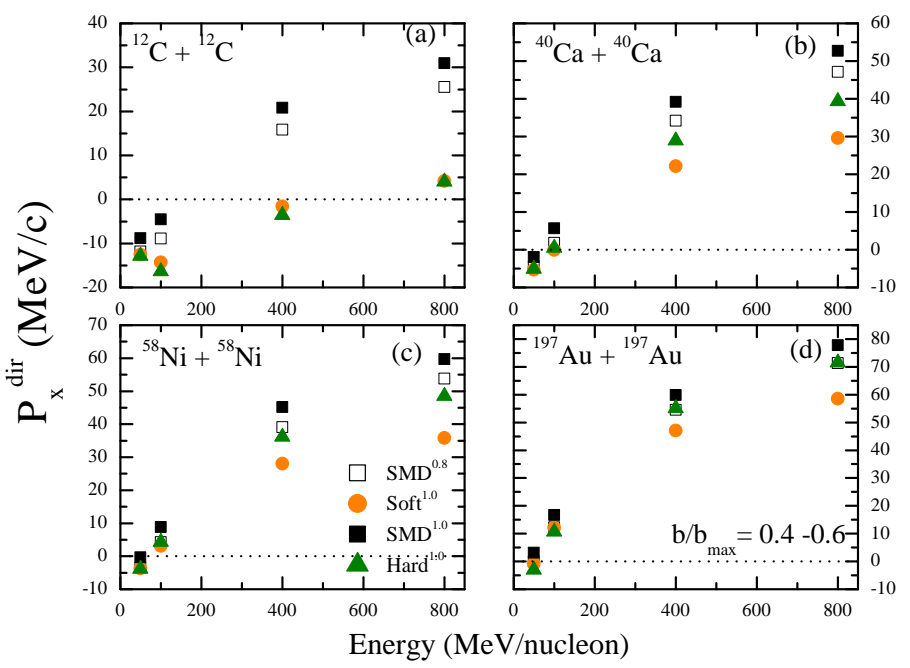

Figure 1. (Color Online) The directed transverse momentum $\left\langle p_{x}^{\text {dir }}\right\rangle$ as the function of incident lab energy for the four different reactions. 
that both momentum dependence of mean field and nucleon nucleon cross-section are repulsive in nature, and hence, results in the increased value of flow. Also the repulsive nature of the momentumdependent forces is more dominant at the higher incident energies for all the cases (compare solid circles and squares). At low incident energies both hard and soft EOS gives almost same flow, as at these energies density achieved is less than normal nuclear matter density, but at high energies hard EOS state leads to more flow as compared to soft EOS as density achieved is more. We also notice that, free cross-section leads to larger flow as compared to the reduced cross-section due to increased in the collisions which are repulsive in nature (compare the open and solid square symbols). Also the role of cross-section remains same at all incident energies.

For mass-dependent analysis of balance energy, we simulated the reactions of ${ }^{12} \mathrm{C}+{ }^{12} \mathrm{C}(\hat{b}=0.4)$, ${ }^{20} \mathrm{Ne}+{ }^{27} \mathrm{Al}(\hat{b}=0.4),{ }^{36} \mathrm{Ar}+{ }^{27} \mathrm{Al}(b=0-2.5 \mathrm{fm}),{ }^{40} \mathrm{Ar}+{ }^{27} \mathrm{Al}(b=1.6 \mathrm{fm}),{ }^{40} \mathrm{Ar}+{ }^{45} \mathrm{Sc}(\hat{b}=0-0.28)$, ${ }^{40} \mathrm{Ar}+{ }^{51} \mathrm{~V}(\hat{b}=0.3),{ }^{64} \mathrm{Zn}+{ }^{27} \mathrm{Al}(b=0-2 \mathrm{fm}),{ }^{40} \mathrm{Ar}+{ }^{58} \mathrm{Ni}(b=0-3 \mathrm{fm}),{ }^{64} \mathrm{Zn}+{ }^{48} \mathrm{Ti}(b=0-2.5 \mathrm{fm})$, ${ }^{58} \mathrm{Ni}+{ }^{58} \mathrm{Ni}(\hat{b}=0-0.28),{ }^{64} \mathrm{Zn}+{ }^{58} \mathrm{Ni}(b=0-2.5 \mathrm{fm}),{ }^{86} \mathrm{Kr}+{ }^{93} \mathrm{Nb}(\hat{b}=0-0.28),{ }^{93} \mathrm{Nb}+{ }^{93} \mathrm{Nb}(\hat{b}=0.3)$, ${ }^{129} \mathrm{Xe}+{ }^{118} \mathrm{Sn}(b=0-3 \mathrm{fm}),{ }^{139} \mathrm{La}+{ }^{139} \mathrm{La}(\hat{b}=0.3)$, and ${ }^{197} \mathrm{Au}+{ }^{197} \mathrm{Au}(b=0-4 \mathrm{fm})$ as per experimental measurements $[4,10,11]$. The reactions are simulated at different fixed incident energies and a straight line interpolation is used to calculate the balance energy. Following the recent analysis [15], liquid drop radius is used for all nuclei except for ${ }^{12} \mathrm{C}$ where experimental radius has been used until stated otherwise. Fig. 2 (a), we display the balance energy as the function of system mass. Circles, triangles, open squares represent the calculations using soft, hard and SMD EOS with $\sigma=0.8 \sigma_{\text {free }}$ respectively, whereas solid squares represent calculations performed by using SMD along with free cross-section. The experimental data is displayed by solid stars along with error bars. The lines follow power law $\propto \mathrm{A}^{-\tau}$. From the Fig. 2(a), we notice that the balance energy calculated using SMD equation of

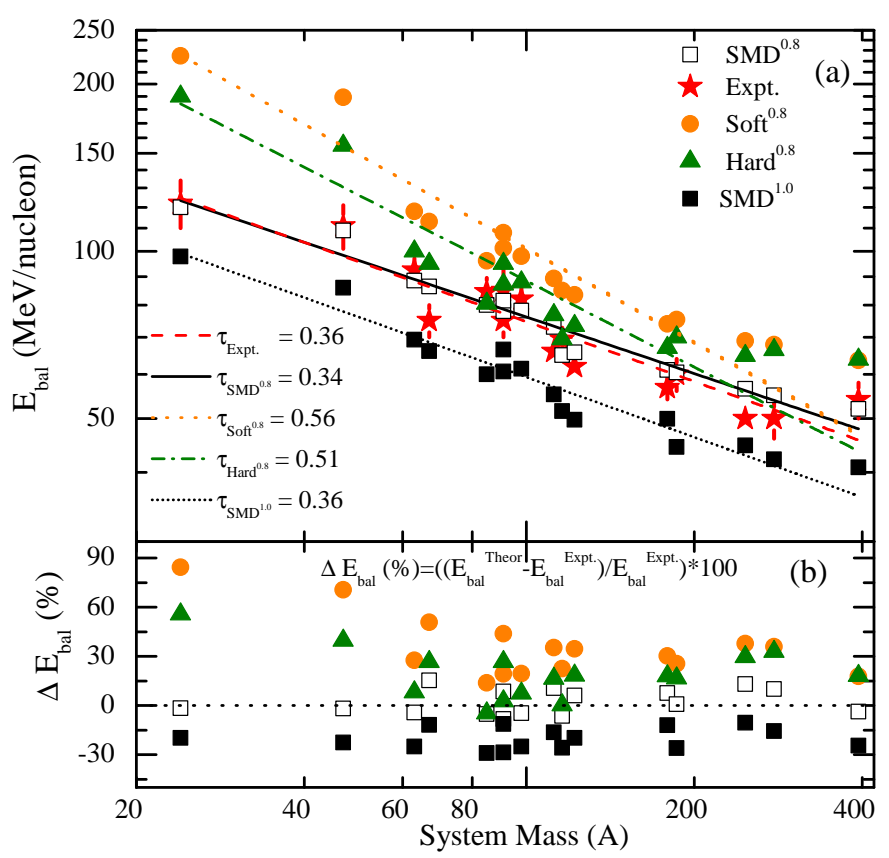

Figure 2. (Color Online) The balance energy (upper panel) and percentage deviation (lower panel) of $\mathrm{E}_{b a l}$ from the experimentally measured $\mathrm{E}_{b a l}$ as a function of system mass. 
the state with reduced cross-section $\left(\sigma=0.8 \sigma_{\text {free }}\right)$ as well as with free cross-section follows almost similar power law dependence, and the value of $\tau$ is also very close to the experimentally determined value. But SMD EOS with free cross-section is not able to reproduce the experimental measured balance energies. In Fig. 2(b) displays the percentage deviation of the balance energy for different sets of EOS and cross-section (mentioned above) from the experimentally measured data. From the figure, we see that deviations are very less for SMD equation of state with reduced cross-section (open squares) compared to the other options. So we came to the conclusion that SMD equation of state along with the reduced cross-section $\left(\sigma=0.8 \sigma_{\text {free }}\right)$ can explain the experimental data well.

In summary, we notice that different equations of states, momentum dependence of nuclear forces and in-medium nucleon-nucleon cross-sections have sizeable effect on the beam energy dependence of the directed flow. Also SMD equation of state with reduced cross-section is the best choice to reproduce the experimental data.

\section{Acknowledgement}

This work has been supported by a grant from University Grant Commission (UGC), Government of India. The author is thankful to Professor Rajeev K. Puri for enlightening discussions on the present work.

\section{References}

[1] J. Jaenicke et al., Nucl. Phys. A 536, 201 (1992).

[2] A. D. Sood and R. K. Puri, Eur. Phys. J. A 30, 571 (2006); A. D. Sood et al., Phys. Lett. B 594, 260 (2004); ibid. C 73, 067602 (2006); ibid. C 69, 054612 (2004).

[3] R. K. Puri et al., J. Phys. G. Nucl. Pat. Phys. 21, 583 (1995); S. Kumar et al., Phys. Rev. C 58, 3494 (1998).

[4] J. Łukasik et al., Phys. Lett. B 608, 223 (2005).

[5] B. A. Li et al., Phys. Rev. Lett. 76, 4492 (1996); R. Pak et al., Phys. Rev. Lett. 78, 1022 (1997); ibid. 78, 1026 (1997).

[6] R. Chugh and R. K. Puri, Phys. Rev. C 82, 014603 (2010); ibid. Int. J. Mod. Phys. E 19, 2009 (2010).

[7] S. Goyal and R. K. Puri, Nucl. Phys. A 853, 164 (2011).

[8] S. Gautam and A. D. Sood, Phys. Rev. C 82, 014604 (2010); S. Gautam et al., Phys. Rev. C 83, 034606 (2011); ibid. C 83, 014603 (2011); ibid. C 86, 034607 (2012).

[9] D. Krofcheck et al., Phys. Rev. Lett. 63, 2028 (1989).

[10] J. P. Sullivan et al., Phys. Lett. B 249, 8 (1990); C. A. Ogilvie et al., Phys. Rev. C 42, R10 (1990); D. Krofcheck et al., ibid. C 46, 1416 (1992); A. Buta et al., Nucl. Phys. A 584, 397 (1995); G. D. Westfall et al., Phys. Rev. Lett. 71, 1986 (1993); D. Cussol et al., Phys. Rev. C 45, 044604 (2002).

[11] Z. Y. He et al., Nucl. Phys. A 598, 248 (1996); D. J. Magestro et al., Phys. Rev. C 61, 021602 (2000); ibid. 014603(R) (2000).

[12] D. Kalkow et al., Phys. Rev. C 48, 1982 (1993); V. de la Mota et al., ibid. C 46, 677 (1992).

[13] S. Kumar et al., Phys. Rev. C 82, 024610 (2010) .

[14] C. Hartnack et al., Eur. Phys. J. A 1, 151 (1998); S. Kaur and R. K. Puri Phys. Rev. C 87, 014620 (2013); S. Kumar et al., Phys. Rev. C 81, 014611 (2010); ibid. 014601 (2010).

[15] R. Bansal, S. Gautam, R. K. Puri and J. Aichelin Phys. Rev. C 87, 061602(R) (2013). 